\title{
Finite Element Analysis of Permanent Magnet Synchronous Motor of Electric Vehicle
}

\author{
Lingling Wang ${ }^{1, a}$, Xin Wang ${ }^{1, b^{*}}$, Yihui Zheng ${ }^{1}$, Lixue $\mathrm{Li}^{1}$, Wei Wang ${ }^{2}$ and Hao \\ $\mathrm{Wu}^{2}$ \\ ${ }^{1}$ Center of Electrical \& Electronic Technology, Shanghai Jiao Tong University, Shanghai, China \\ ${ }^{2}$ Yanbian Grid Company Grid Company of Jilin Province, Yanbian 138000, China \\ awllizsx@163.com, bwangxin26@sjtu.eu.cn \\ *corresponding author: wangxin26@sjtu.edu.cn
}

\begin{abstract}
Keywords: Electric vehicle, Permanent magnetic synchronous motor, Electromagnetic fields, Torque, Finite element analysis

Abstract. In this paper, finite element analysis is used to analyze the torque of Permanent Magnet Synchronous Motor (PMSM) of electric vehicle. Firstly, a 2D model of the PMSM is made. By forming magnetic field lines and flux density distribution of the PMSM with no-load condition, the accuracy of the model can be verified. Secondly, torque fluctuation curve of the PMSM with rated load is analyzed. It shows that the torque of the motor meets the stability requirements. Finally, by setting different current stimulations, analyzing the change of the motor output torque, it can be concluded that the relationship between motor torque and current amplitude is nonlinear, which lays a foundation for the operation optimization of the PMSM used in electric vehicle.
\end{abstract}

\section{Introduction}

The enormous emission of traditional automotive exhaust is forcing human beings to pursue cleaner energy. The appearing of electric vehicles can solve this problem[1]. As one of the key components for electric vehicle, the driving motor needs to meet higher requirements. In order to ensure the excellent performance of electric vehicle, it should have high speed and wide speed range, as well as a large starting torque and high efficiency.

Since Permanent Magnet Synchronous Motor (PMSM) is one of the most used motor type of electric vehicle, analyzing its output torque is of great importance. In [2], the relationship between permanent magnet position and output torque is analyzed, which can optimize the PMSM structure. Reference [3] used the genetic algorithm for integrated optimization of the rotor magnetic pole structure, and the cogging torque can be reduced as well as the torque fluctuation. In [4], motor torque is analyzed from the point of current space Angle.

Finite element analysis is used in this paper to analyze the torque of PMSM of electric vehicle. Firstly, a 2D model of the PMSM is made. Secondly, torque fluctuation curve of the PMSM with rated load is analyzed. Finally, by setting different current stimulations, the relationship between motor torque and current amplitude is observed. The study can lay a foundation for the operation optimization of the PMSM used in electric vehicle.

\section{D Finite Element Modeling of PMSM}

The Process of Modeling. Finite element analysis method is a method using mathematical approximation method for simulating real physical system structure. It can be suitable for all kinds of complicated structure, has the characteristic of high precision, and is the effective means of engineering analysis. The idea of finite element analysis method is to make the overall structure discrete, using limited units to represent a complex object. The units are connected by finite number of nodes. Finally according to the boundary conditions of the model, a comprehensive solution can be found. Here, Ansoft software is used for modeling and solving, and the procedure contains 7 steps: (1) 
Establish PMSM's geometry physical model; (2) Set component's material properties; (3) Determine the boundary conditions; (4) Mesh partition; (5) Set motion parameters; (6) Solve motor's operational performance; (7) Postprocessing.

A PMSM used by certain electric car with the rated power of $7.5 \mathrm{~kW}$ is selected as the simulation object, the basic parameters of the motor are shown in Table 1. The 2D finite element model is set up according to the size of the PMSM, then completing the settings of windings and material properties and setting the magnetic field as a nonlinear constant one, the 2D finite element model can be designed in Fig. 1.

Table 1. Structure parameter of PMSM

\begin{tabular}{|c|c|c|c|}
\hline Power & $7.5[\mathrm{~kW}]$ & Pole-pairs & 8 \\
\hline Inner diameter of stator & $162[\mathrm{~mm}]$ & Outer diameter of stator & $269[\mathrm{~mm}]$ \\
\hline Inner diameter of rotor & $110[\mathrm{~mm}]$ & Outer diameter of rotor & $160[\mathrm{~mm}]$ \\
\hline Number of stator slots & 48 & Number of permanent magnets & 16 \\
\hline
\end{tabular}

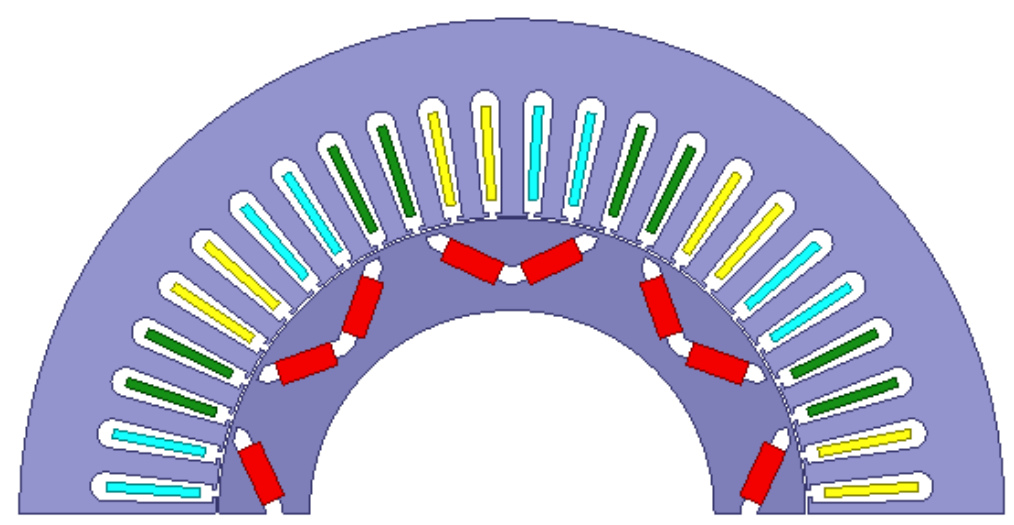

Fig. 1. 2D model of PMSM based on Ansoft software

Because of the symmetry of the structure, it can analyze just $1 / 8$ of the whole model to decrease the number of finite element and save the simulation calculation time. After setting boundary conditions of the geometric model and the meshing, the quality of the meshing is directly related to the accuracy of the calculation results. Here Ansoft software adopts adaptive meshing method, automatically modifies the gridding size by repeated iteration and finally forms a reasonable meshing.

Solution of the Model. After the meshing, the electromagnetic calculation of the PMSM is needed. By electromagnetic field theory, the process of analyzing electromagnetic field is to solve Maxwell's equations, the detailed solving procedure can refer to literature [5].

\section{Electromagnetic Field Analysis of PMSM}

Electromagnetic Field Analysis of PMSM with No Load. After completing the meshing, the approximate solutions of every solution domain and the magnetic field distribution can be received through the study of the finite element calculation of each node. Ultimately, through Ansoft software's powerful post-processing function, PMSM's magnetic field lines and flux density distribution under no-load state can be shown in Fig. 2.

In the case of no-load and no three-phase current in the three-phase stator winding, the stator does not create magnetic field. So the no-load magnetic field is totally created by the rotor's permanent magnets. To analyze the no-load magnetic field is to analyze the interaction of permanent magnet and stator and rotor magnetic circuit. From Fig. 2, it can be seen that the silicon steel sheet near permanent magnet is highly saturated, which is caused by the permanent magnets[6]. 

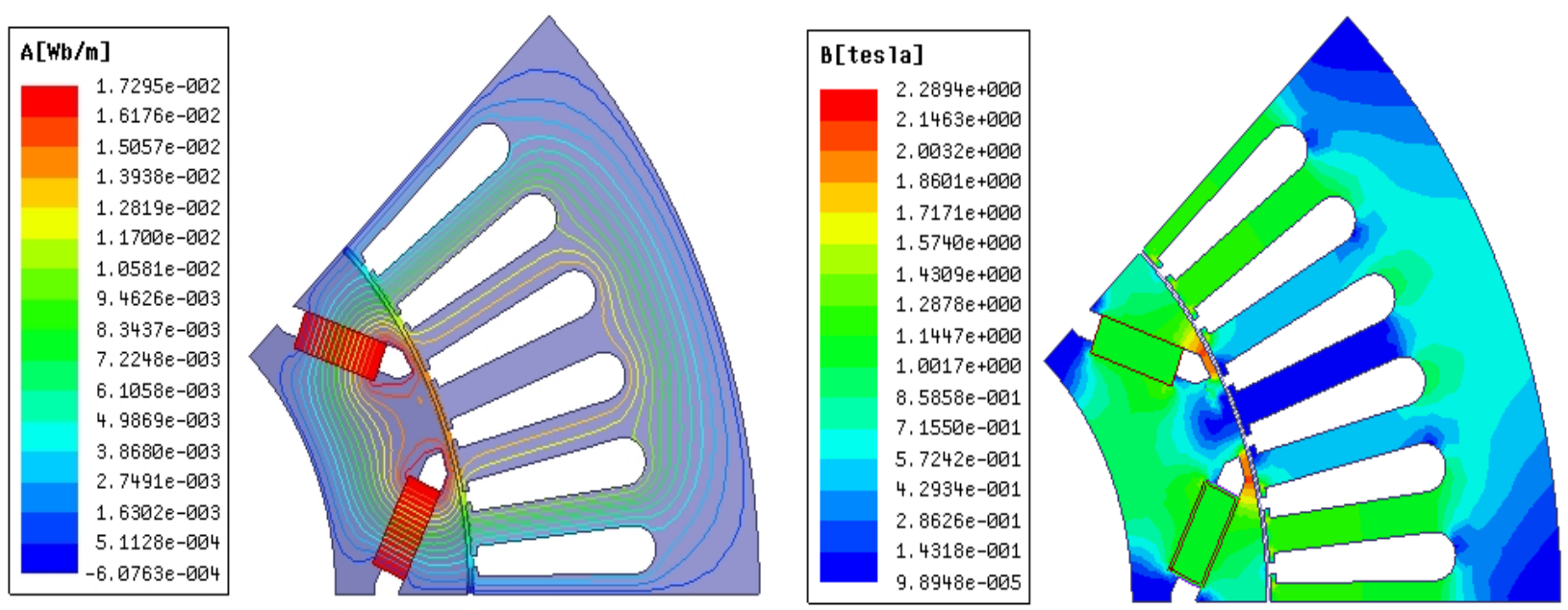

Fig. 2. No-load distribution of magnetic field lines and flux density distribution of PMSM

Torque Analysis of PMSM. Torque curve of PMSM with rated load is shown in Fig. 3. It can be seen that the lowest value of the torque is about $157 \mathrm{~N} . \mathrm{m}$, the highest value is about $188 \mathrm{~N} . \mathrm{m}$, the average torque is about $173 \mathrm{~N} . \mathrm{m}$, and the torque fluctuation is less than $10 \%$. So it can maintain the stationarity of electric car when running.

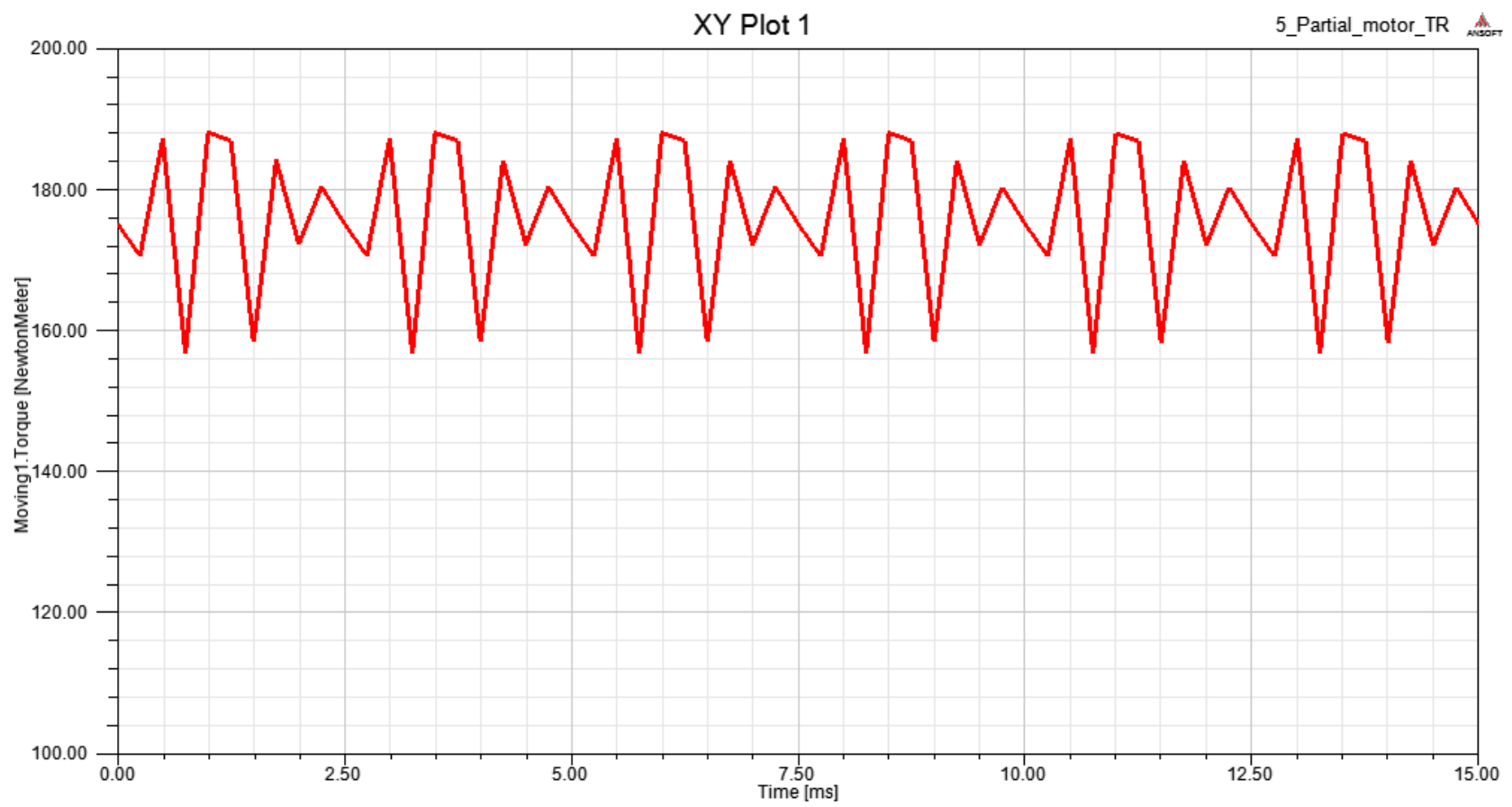

Fig. 3. Torque curve of PMSM with rated load

Current Impact on the Torque of PMSM. In the motor model, the excitation is three-phase alternating current. By changing the current amplitude, the change of PMSM's output torque can be observed. Furthermore, the relationship between PMSM's output torque and the amplitude of the current provided can be observed. For this purpose, we change the parameter in Ansoft transient simulation, ensure the amplitude of the current range from 10A to 500A. A series of torque waveforms can be obtained, and by using Ansoft software's curve fitting function, the relationship between torque and the current amplitude is shown in Fig. 4. It can be easily seen that the relationship between motor torque and current amplitude is nonlinear. 


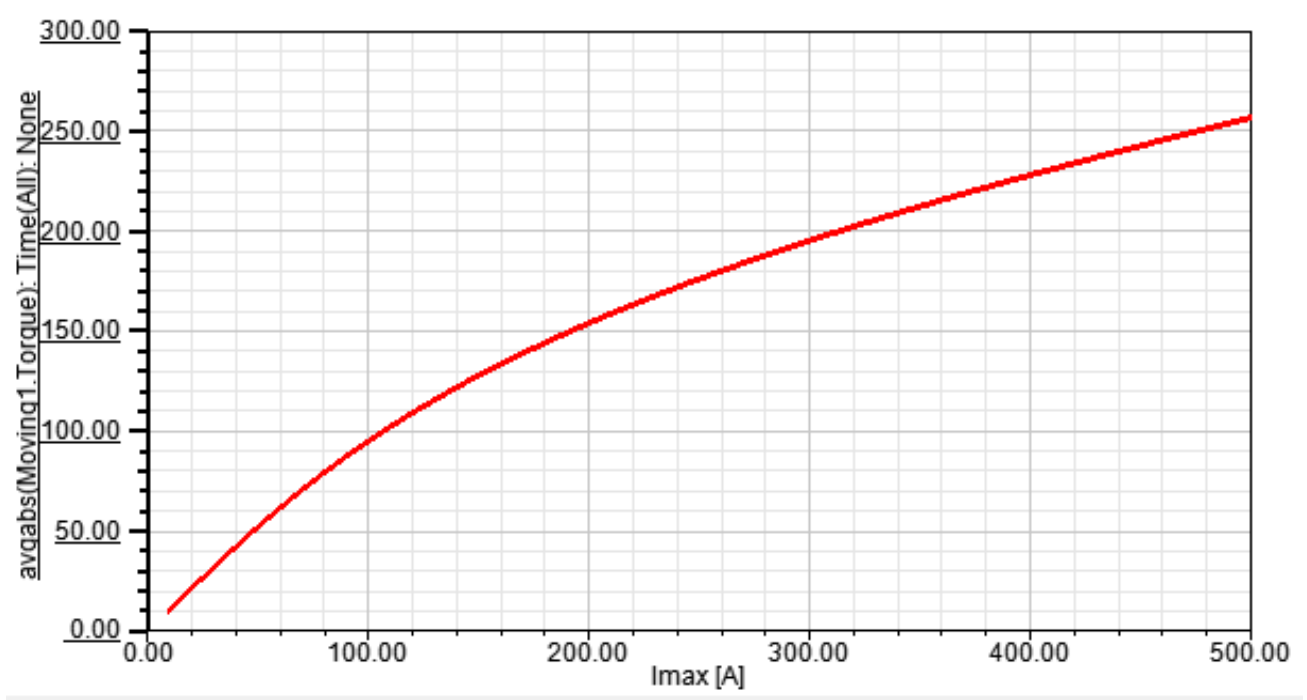

Fig. 4. Curve of motor torque and current amplitude

\section{Conclusions}

The emergence and development of electric car can solve the problem of the enormous emission of traditional automotive exhaust. Ansoft software is used to build PMSM model of an electric car, then related theory of electromagnetic field and finite element analysis are combined to analyze the PMSM model, then the distribution of electromagnetic field and the cogging torque curve of the built PMSM model with rated load is analyzed, finally the accuracy of the design of the motor is verified, different motivations are set to observe the change of the motor output torque, it can lay a foundation for the operation optimization of the PMSM.

\section{Acknowledgements}

This work is supported by the Key Project of NSFC (No. 61533012), the Shanghai Natural Science Foundation (14ZR1421800), the State Key Laboratory of Synthetical Automation for Process Industries.

\section{References}

[1] T.C. Wang, Y. Dai, S.M. Cui, Research status of permanent magnet synchronous motor of electric car, Micromotors, 38 (2005) 55-57.

[2] Q. Fu, A.M. Wang, L.Wei, Research on magnetic cross-coupling impacts on interior permanent magnet machine controls with wide speed operation, Micromotors, 44 (2011) 40-45.

[3] X.D. Xu, X.Z. Xu, H.F. Feng, Present study and prospect of the cogging torque comprehensive reducing methods of PM motors, Micromotors, 42 (2009) 64-68.

[4] Z. Q. Zhu, C. C. Chan. Electrical machine topologies and technologies for electric hybrid, and fuel cell vehicles, IEEE Vehicle Power and Propulsion Conference(VPPC), Harbin China, (2008) 3-5.

[5] X.T. Duan, X.Y. Zhang, J. Zhang, Electromagnetic field calculation and analysis of doubly-fed wind power generator based on the finite element method, Power System Technology, 36 (2012) 231-236.

[6] Y.H. Jin, H.W. Wang, Optimize flux density of air gap of permanent magnet synchronous motor based on Ansoft, Micromotors, 46 (2013) 83-87. 